\title{
La Ley de la tendencia descendente de la tasa de ganancia y su validez explicativa de las crisis económicas. La nueva interpretación temporalista
}

\author{
The Law of Tendency of the Rate of Profit to Fall, \\ and their explanatory validity of economic crises. \\ The new temporalist approach
}

\begin{abstract}
Sergio Martín Fernández
Economista e Historiador económico, graduado en Economía por la Universidad Autónoma de Madrid y posgraduado en Historia Económica por la Universitat de Barcelona.

http://orcid.org/0000-0002-8792-4534

sergio.martinf.91@gmail.com

Fecha de recepción: 05 de noviembre de 2015

Fecha de aceptación: 11 de febrero de 2016
\end{abstract}

Sugerencia de citación: Martín, S. (2016). La Ley de la tendencia descendente de la tasa de ganancia y su validez explicativa de las crisis económicas.

La nueva interpretación temporalista. tiempo\&economía, 3(1), 39-58, doi: http://dx.doi.org/10.21789/24222704.1094

\section{Resumen}

Este trabajo es una revisión de los debates clásicos y actuales sobre la Ley de la tendencia descendente de la tasa de ganancia y su validez como teoría explicativa de las dinámicas del capitalismo. El texto trata de responder a la consistencia o no de la Ley, puesta en duda durante décadas, a través de la presentación de la nueva interpretación temporalista (TSSI) y la adecuación de esta nueva interpretación marxista para explicar las crisis económicas en el capitalismo. 
Palabras clave: Marxismo,crisis, tasa de ganancia, LTDTG, TSSI.

Códigos JEL: B14, B24, D46, E01, G01

\begin{abstract}
This work is a revision of the classic and current debates on the Law of the Tendency to Fall in the Rate of Profit, as an explanatory theory of the dynamics of Capitalism. The text attempts to check the consistency of this controversial Law, through the presentation of the new temporalist interpretation (TSSI), and the adequacy of this new Marxist interpretation to explain the economic crisis in the Capitalism.
\end{abstract}

Keywords: Marxism, Crisis, Rate of Profit, LTPRF, TSSI.

JEL Codes: B14, B24, D46, E01, G01 


\section{Introducción}

Este trabajo presenta una síntesis sobre la historia teórica en torno a la Ley de la tendencia descendente de la tasa de ganancia (LTDTG), ${ }^{1}$ que incide en los debates más recientes que han dividido a la escuela marxista sobre el origen y desarrollo de las crisis capitalistas. Este artículo parte de considerar esta Ley como el elemento principal de la teoría marxista de las crisis capitalistas. El principal objetivo será evaluar, a través de la revisión de las críticas y los debates que ha suscitado dicha ley, la validez de la LTDTG frente a las críticas de inconsistencia como teoría explicativa de las crisis capitalistas.

En este documento se partirá del planteamiento original de Karl Marx, seguido por el repaso de los primeros críticos, y se finaliza con los debates actuales surgidos en torno al renovado enfoque de la Temporal Single-System Interpretation (Interpretación Temporal del SistemaSimple o TSSI).

El contexto actual está marcado por el derrumbe de las economías más desarrolladas en 2009, el posterior shock en todas las regiones mundiales, incluidos los países BRICS y nuevos BRICS, y la prolongada depresión del capitalismo europeo, lo que ha expuesto a un ambiente de fuertes críticas a la teoría mainstream de la economía; críticas llevadas a cabo desde diferentes frentes, pero con mayor énfasis desde los enfoques heterodoxos, que habían sido relegados de las facultades, de las publicaciones académicas y de la política económica.

Así, la crisis surge como una anomalía, en términos de Kuhn y Lakatos (Burawoy, 1990), que altera el paradigma dominante (neoclásico), la cual ha provocado una crisis en este y posibilitado que los enfoques heterodoxos iniciasen una "revolución" contra el enfoque dominante y sus fallas (Burawoy, 1990), que nos traslada al panorama actual de competencia entre escuelas, que reclaman a sus núcleos duros para sustituir al paradigma en crisis, retornando a autores como Adam Smith, Ricardo, Marx o Keynes.

Este debate aparentemente teórico, también ha llegado a las aulas de las facultades de Economía, en las cuales profesores y alumnos han reclamado la incapacidad de los actuales programas de estudio para formar economistas que tengan las herramientas necesarias para comprender el funcionamiento de la economía capitalista. ${ }^{2}$ Tal crítica está basada en la monoparadigmacización de los programas de estudio y de la producción científica dentro de las revistas de mayor impacto. En parte, este trabajo se enmarca dentro de este movimiento de apertura en los planes de estudios e investigación en la economía, a través del estudio de uno de los enfoques heterodoxos que ha sido apartado de las facultades, el enfoque marxista.

Partiendo de este contexto y unido al clima social derivado de la crisis económica que lleva a la sociedad a pedir respuestas y soluciones a problemas como el desempleo, el hambre o la pérdida de sus viviendas, este trabajo pretende aportar algo de claridad sobre la interpretación

1 De ahora en adelante, en el texto aparecerá por sus siglas LTDTG.

2 Se han formado distintos grupos de estudio y de soporte para la transformación de los planes académicos, que recogen el testigo del Movimiento Postautista de las universidades de París. Destacan la Post-Crash Economics Society de Manchester, la Rethinking Economics o el Netzwerk Plurale Ökonomik. Estos y otros grupos y asociaciones de estudiantes formaron la ISIPE (International Student Initiative for Pluralism in Economics),también apoyada por académicos como Alejandro Nadal, Carlos Berzosa, Oscar Carpintero, T. Piketty, S. Keen o Paul Davidson. 
marxista de las crisis económicas, y de esta forma abrir el debate sobre la capacidad de esta para entender el capitalismo.

\section{LA LEY DE LA TENDENCIA DESCENDENTE DE LA TASA DE GANANCIA Y SUS CRÍTICOS}

\section{La Ley}

En el capítulo XIII de El capital, Marx define la Tasa de Ganancia como la relación entre la Plusvalía y el Capital global invertido, es decir, la suma del capital constante y el capital variable. De este capítulo extrae la ley que define el funcionamiento interno de las dinámicas de acumulación del capitalismo:

La misma cuota de plusvalía, con un grado invariable de explotación del trabajo, se expresaría en una cuota decreciente de ganancia, ${ }^{3}$ puesto que al aumentar su volumen material, aunque no siempre en la misma proporción, aumenta también el volumen del capital constante y con ello, del capital global. (Marx, 2007 [1894], p.278)

Por tanto, significa que el objetivo principal del capitalista es la necesidad de valorización del capital. Lo que implica la búsqueda de métodos más productivos que permitan incrementar la masa de ganancias, esto es, alzar la Plusvalía, siendo esta el trabajo generado por los trabajadores y no remunerado, del que se apropian los capitalistas. Sin embargo, este camino que toman los capitalistas no tiene por qué ser racional, en tanto que una de sus consecuencias es el incremento del capital constante respecto al capital variable, según lo definió Marx, como el aumento de la composición orgánica del capital (COC).

Este proceso emana de la Teoría del Valor Trabajo (TVT) ${ }_{1}^{4}$ base esencial de la teoría económica de Marx. Según esta teoría, la introducción de una nueva técnica de producción implica la expulsión de una parte de los trabajadores, que pasan a ser fuerza de trabajo ociosa. Esto produce dos efectos antagónicos sobre la Tasa de Ganancia: uno positivo, el aumento de la Tasa de Plusvalía, y otro negativo, el incremento de la COC. Marx (2007) sostuvo que el efecto de la contracción relativa del capital variable era mayor que el incremento de la plusvalía, y que, por lo tanto, la Tasa de Ganancia caería, no de una forma absoluta y directa, sino de manera tendencial. La búsqueda de una explicación de por qué esta Tasa no cae más rápidamente es la pregunta esencial para Marx, puesto que la tendencia ya había sido anteriormente resaltada

3 Siguiendo a la mayoría de las traducciones realizadas por castellanoparlantes, en el texto, a la Rate of Profit se la denominará "tasa de ganancia", a pesar de que en la traducción utilizada de El capital se decantan por cuota de ganancias.

4 Explicar la teoría del valor de Marx, aunque simplemente fuera un resumen, implicaría un artículo en sí mismo. No obstante, se recomienda a los interesados o curiosos del tema algunas referencias bibliográficas: Capítulo III, en Guerrero (2008); Capítulo II, en Shaikh (1990), o Capítulo II, en Kliman (2007), así como la propia obra original de Marx, para poder comprenderla. 
tanto por Smith como por Ricardo. Son por tanto los argumentos y las explicaciones sobre la forma, el tiempo de caída y su recuperación los que hacen diferentes y determinantes las observaciones de Marx.

Si bien es cierto que Marx no descubrió que el capitalismo engendrara una tendencia a la caída de la Tasa de Ganancia, sí argumentó una explicación coherente y formuló una teoría que la explicaba: la Ley de la Tendencia Descendente de la Tasa de Ganancia (a partir de ahora, en el texto aparecerá como LTDTG), y los frenos que actuaban contra la misma. Marx señaló cinco causas principales que dentro del propio sistema capitalista permitían contrarrestar temporalmente la caída de la Tasa de Ganancia:

- El aumento del grado de explotación: "El grado de explotación del trabajo, la apropiación del Plustrabajo y de Plusvalía se elevan sobre todo mediante la prolongación de la jornada de trabajo y la intensificación del trabajo" (Marx, 2007 [1894], p.305). La reducción del salario por debajo de su valor, lo cual genera un incremento de la Plusvalía, que pudiera sobrepasar el aumento de la COC.

- La formación de un excedente de población que no tiene la posibilidad de vender su fuerza de trabajo. Es decir, la emergencia de una superpoblación relativa, debida a la mayor velocidad con la que se presenta la productividad del trabajo con respecto al nacimiento de nuevos proyectos para la inversión de capital. Esta característica es inseparable del desarrollo de las fuerzas productivas dentro del capitalismo.

- El comercio exterior, que permite reducir el coste del capital constante y la inversión en el exterior, en países menos avanzados. Esto posibilita elevadas Tasas de Ganancias, que pueden complementar las caídas.

Sin embargo, Marx determinó que la acción de estas cinco causas no puede contrarrestar la Ley de la baja de la Tasa de Ganancia, solo la frenan: "Las mismas causas que producen la baja de la cuota general de ganancia provocan reacciones obstaculizadoras, amortiguan y en parte paralizan esta baja. No anulan la ley, pero atenúan sus efectos" (Marx, 2007 [1894], p.315).

Llevándole a concluir que la tendencia de la Tasa de Ganancia en el tiempo sería a la baja, y que esta caída provocaría recurrentes crisis de valorización del capital, impidiendo la continuidad del proceso de acumulación. De donde emanarían las crisis capitalistas, al no poder recuperar lo invertido, dificultando las inversiones del periodo siguiente, que explica de la siguiente manera:

Como la cuota de valorización del capital global, la cuota de ganancia, es el acicate de la producción capitalista (lo mismo que la valorización capitalista es su única finalidad), su baja amortigua la formación de nuevos capitales independientes y se presenta así como una amenaza para el desarrollo del proceso capitalista de producción: fomenta la superproducción, la especulación, las crisis, el exceso de capital al mismo tiempo que exceso de población [...] Mas lo importante en su horror [hablando de Ricardo] a la cuota descendente de ganancia es la sensación de que el modelo capitalista de producción halla un límite en el desarrollo de las fuerzas 
productivas que nada tiene que ver con la producción de la riqueza en cuanto tal; y esta barrera peculiar acredita la limitación y el carácter puramente histórico, transitorio, del modo capitalista de producción, acredita que no es ningún modo absoluto de producción para la producción de riqueza, sino que más bien entra en conflicto con su desarrollo ulterior al llegar a cierta fase. (Marx, 2007 [1894], p.318)

Por lo tanto, la propia necesidad del capital de ampliar la plusvalía, unida a la lucha competitiva entre capitales, lleva a que estos tengan que aplicar recurrentemente nuevas tecnologías. Pero estas, a su vez, producirán una caída de la Tasa de Ganancia. Lo que supondrá una presión competitiva cada vez mayor por las ventas en los mercados, hasta llegar al estallido de procesos de sobreproducción y crisis de destrucción o desvalorización del capital existente.

Solo mediante la desvalorización del capital existente es capaz el capitalismo de hacer frente a las crisis por la caída de la tasa de ganancia. Comenzando un nuevo ciclo de acumulación de capital, o lo que es lo mismo, un nuevo ciclo vicioso. Siendo: "[...] el verdadero límite de la producción capitalista el propio capital [...]" (Marx, 2007 [1894], p.329)

\section{Las críticas a la teoría de Marx}

Las críticas realizadas a Marx y a la LTDTG son muy numerosas y diversas, por lo que se presentarán las más importantes y se clasificarán en dos grupos para facilitar la comprensión de las mismas. Estos dos grupos se dividirán entre las críticas a la TVT y las críticas a la formulación de la LTDTG.

Dentro del primer grupo de críticas habría que destacar tres autores que escribieron entre los siglos XIX y XX, Dmitriev, Böhm-Bawerk y Bortkiewicz. Los tres autores rechazaron que el valor de las mercancías esté determinado únicamente por el trabajo humano, y que por lo tanto la ganancia esté basada en la sustracción de trabajo no remunerado por los capitalistas. Existen diferencias entre los tres autores, y en cómo influyeron en los posteriores críticos.

Dmitriev sostenía que el valor de las mercancías no dependía de ninguna relación especial del trabajo utilizado en la producción. Este autor procede a demostrarlo por medio de un modelo complejo, que carece de trabajo humano y en el cual la maquinaria construye nueva maquinaria, dando un soporte a lo que posteriormente también defendería Okishio, que la Tasa de Ganancia siempre sería positiva (cf. Kliman, 2007). Por otra parte, Böhm-Bawerk y más tarde Bortkiewicz ahondaron en la existencia de un problema en la transformación de valores en precios en el texto de Marx, que para ambos autores significaba que la teoría del valor y el cálculo de la Tasa de Ganancia de Marx eran claramente inconsistentes. Bortkiewicz sería el primer autor en intentar elaborar una corrección al trabajo de Marx, soporte también para un número creciente de autores marxistas posteriores, los Neo-marxistas, sobre todo después de que Sweezy y Baran la popularizaran (cf. Kliman, 2007).

Sweezy y Baran crearon una revista académica y una escuela a su alrededor, siendo esta la primera gran divergencia en la interpretación marxista de las crisis, e influyeron en la siguiente generación de marxistas que nació dentro de las universidades, y que se vieron atraídos por 
las técnicas y los enfoques nuevos provenientes de Cambridge, los llamados Neo-ricardianos o Sraffiano. ${ }^{5}$ Esta nueva generación de académicos empezaría a utilizar el esquema BortkiewiczSraffa para replantear toda la teoría marxista, aceptando de partida la inconsistencia en Marx.

Poco a poco, la nueva teoría marxista enseñada en las facultades y escuelas de Economía tenía más de Keynes y de Sraffa que del Marx original, en la que se daban por sentado las inconsistencias tanto en su Teoría del Valor como en su interpretación del origen de las crisis económicas. A estos autores se les sumaron otros economistas de clara formación neoclásica como Samuelson, quien adaptando la corriente de la Whiggish History formuló un planteamiento evolucionista del conocimiento económico, según el cual toda teoría presente supera las teorías predecesoras. Esta forma de plantear el conocimiento económico era muy oportuna, pues le permitía asegurar que la teoría del valor y los planteamientos de Marx dejaron de tener solidez teórica al ser corregidos y mejorados por sus sucesores, siendo lo correcto utilizar los modelos sraffianos (cf. Kliman, 2014; Freeman, Chick y Kayatekin, 2014).

En el segundo grupo cabría resaltar a Joan Robinson. Como teórica keynesiana y defensora de la teoría de la demanda efectiva, tuvo una fuerte repercusión con su crítica a la Teoría Marxista (Robinson, 1968). Esta economista expuso su crítica contra la LTDTG a través de dos puntos. En el primero y más importante, Robinson asegura que Marx presenta una contradicción entre supuestos y conclusiones, al utilizar una tasa constante de explotación a la hora de calcular la Tasa de Ganancia, siendo esta una primera incongruencia de la Ley. Debemos señalar el error que comete J. Robinson al no haberse detenido en la lectura de El capital, puesto que Marx no sostiene que la explotación sea constante; todo lo contrario, expone que cualquiera de las formas de acrecentarla no puede contraponerse a la creciente COC.

El segundo reproche por parte de esta autora a Marx se centra en magnificar una de las causas contrarrestantes, en concreto, el abaratamiento del capital constante, que neutralizaría la caída de la Tasa de Ganancia. Esta reflexión ha dado origen a una de las teorías que más seguidores tiene entre los críticos de Marx, la Profit squeeze. Los autores que la sostienen inciden en los salarios como única variable capaz de hacer caer la Tasa de Ganancia, lo que provocaría un estrangulamiento en las ganancias: "Otros críticos de la ley reconocen caídas de la tasa de ganancia, pero interpretan que ese fenómeno no obedece a contradicciones internas de la acumulación. Frecuentemente atribuyen la declinación a un comportamiento ascendente de los salarios" (Katz, 2010, p.101). Hay que entender que estos autores no niegan que pueda caer la Tasa de Ganancia, pero sí formalizan su explicación de manera muy diferente a la original y se oponen a la explicación de la LTDTG.

Tres son las diferentes visiones que sostiene la Profit squeeze:

a) La visión de los teóricos de la regulación, que, como Goldstein (Katz, 2010), recalcan la influencia de las subidas salariales en la productividad y la rentabilidad.

5 Hay que hablar de sraffianos,y no de Sraffa, ya que, como expone Astarita (2011): "Es que si bien Sraffa no criticó a Marx, muchos sraffianos plantearon que, a partir de Sraffa, podía demostrarse que la teoría del valor trabajo no es necesaria para determinar los precios o demostrar la existencia de explotación". Según Roncaglia (Astarita, 2011), sería más acertado denominarlos ultrasraffianos.

tiempo\&economía

Vol. 3 N $^{\circ} 1$ - I semestre de 2016

p. 45 
b) Por otra parte, la visión de los que explican el comportamiento de la economía a través del reparto de la renta del país entre salarios y beneficios: el reparto de la tarta. Esta posición es la más politizada, por su capacidad práctica en el terreno social.

c) Por último, la teorización más difundida, y a su vez la crítica más popular a la Teoría de las Crisis à la Marx: el Teorema de Okishio (TO). Este teorema se presenta con un modelo sin capital fijo y sostiene que la Tasa de Ganancia, ante un incremento de la productividad, solo puede crecer, y concluye que la única posibilidad de que la caída de la Tasa de Ganancia se produjera tendría que provenir del incremento del valor del capital variable, es decir, del incremento de los salarios. Esta teorización ha sido la principal crítica expuesta contra la LTDTG, popularizada por autores como Roemer, el más reconocido de ellos y quien ha generado modelos matemáticos a partir de las mismas condiciones que Okishio (cf. Kliman, 2007); ${ }^{6}$ y por otros representantes de esta visión como Paul Sweezy y Maurice Dobb (Kliman, 2007), quienes defienden que el capitalista tiende a incrementar la mecanización del proceso para la sustitución de trabajo por un previo aumento de los salarios que habría reducido la Tasa de Ganancia.

EITO, de la forma planteada por Sweezy y Dobb, permite abrir un debate sobre la elección de las técnicas de producción, puesto que Okishio sostiene "que los capitalistas nunca escogerían emplear una técnica de producción que disminuyera su tasa de ganancia" (denominada por Shaikh "criterio de optimización", cf. Shaikh, 1990). En Marx se opone una elección no voluntaria, debido a la guerra competitiva que predomina en el sistema capitalista, por lo que el capitalista no puede seleccionar la tecnología que al final del proceso le permitirá obtener la mayor ganancia, sino la que le permitirá aumentar la productividad.

Las críticas que hemos presentado en este apartado se muestran esenciales para comprender el ostracismo al que se ha condenado durante décadas a la teoría marxista original, en parte motivado por la relativa inexistencia de una interpretación que pretendiera replicar las críticas a Marx. Por otro lado, que las críticas tuvieran un peso argumentativo fuerte junto a una modelización muy suntuosa y matematizada atrajo la aceptación en la academia de esta revisión de Marx, que aportó un soporte esencial para su difusión.

Expongo estas críticas hasta los años ochenta porque es en esta década cuando los debates toman otro formato, por la aparición de un grupo de autores que sostiene un planteamiento al que podríamos denominar ortodoxo respecto a la teoría de Marx.

6 Aparte de estas tres grandes líneas cabe reseñar el debate entre Lenin y Tugan-Baranowsky sobre la importancia del consumo para la acumulación capitalista. Este último acabaría formalizando una teoría sobre las crisis que aún tiene numerosos continuadores: la teoría de la desproporcionalidad (Shaikh, 1990). También cabe señalar la gran cantidad de publicaciones marxistas y autores que han dedicado tiempo y esfuerzo importantes a la teoría subconsumista, como Rosa Luxemburgo, Paul Sweezy y Paul Baran. 


\section{LA TEMPORAL SINGLE SYSTEM INTERPRETATION}

\section{¿Qué es la Temporal Single System Interpretation?}

En la década de los ochenta surge una nueva interpretación de la teoría marxista bajo el nombre de Temporal Single System Interpretation, un grupo de autores que formulan sus trabajos con el objetivo de defender la validez y consistencia de los argumentos de Marx (TVT) y de las conclusiones que extrajo (LTDTG). La interpretación hermenéutica sobre los textos de Marx los caracteriza y les permite integrar una serie de supuestos dentro de la Teoría de Marx, con el objetivo de confrontar las críticas que expusimos en el punto anterior.

La premisa innovadora que les permite resolver el problema de transformación gravita en torno al tiempo, entendido como la existencia de un lapso temporal entre el momento de introducir los insumos y el instante de salida y realización del output. En este lapso de tiempo los precios y, por tanto, los valores que los determinan pueden haberse visto alterados. Este supuesto de temporalidad les da el nombre a sus defensores, agrupados habitualmente bajo el término temporalistas.

Dentro de la interpretación temporal hay un grupo de autores donde se destacan Andrew Kliman, Alan Freeman, Guglielmo Carchedi, Michele Naples o M. Roberts, quienes colaboran asiduamente en numerosas publicaciones. Por otra parte, podríamos considerar un segundo grupo, compuesto por Rolando Astarita, Jose.A. Tapia, Diego Guerrero, Anwar Shaikh o Michael Husson, que aunque no defienden por completo el argumentario de los temporalistas, , sí defienden los trabajos de estos autores. En tanto que permiten un resurgimiento de la Teoría de las Crisis desde la óptica marxista y amplían los debates en torno a la TVT y la Tasa de Ganancia.

Podemos relacionar a los temporalistas con un marxismo ortodoxo, en tanto que su interpretación se centra en defender la consistencia y validez de los argumentos y la teoría propuestos por Marx. Al mismo tiempo, en el prefacio de la obra más completa de esta escuela, Freeman y Carchedi (1995) aclaran que no pretenden constituir un dogma, ya que ellos no persiguen asegurar la plena consistencia de la Teoría, sino procurar establecer los errores de los autores que hasta la actualidad defienden la "inconsistencia" de los elementos fundamentales del trabajo de Marx.

Los temporalistas atribuyen el conjunto de errores e incoherencias contenidos en los trabajos críticos respecto a Marx a una mala interpretación de lo que verdaderamente Marx escribió en sus obras, principalmente en El capital. Los autores de la TSSI advierten en esos trabajos dos errores fundamentales: el primero y más importante, una visión equivocada sobre la formación del cuerpo teórico de Marx, que los lleva hacia un problema de circularidad, al intentar formular una teoría marxista sin Marx (Freeman, 2000), en tanto que aceptan parte de las conclusiones de este autor, pero a su vez desechan el tronco principal en el que se sustentan todas, la TVT. Esta premisa conduciría a los críticos a aceptar unas conclusiones erróneas, al ser formuladas a partir de una base teórica equivocada. 
El segundo error consistiría en calcular la Tasa de Ganancia por medio de la Plusvalía, y posteriormente interpretar que las crisis son originadas por los incrementos de los salarios. Esta doble argumentación implica una incongruencia, que dejaría sin explicación tanto la Tasa de Ganancia como su signo. Kliman aclara que los errores de inconsistencia teórica que se le atribuían a Marx no estarían en los escritos originales pero sí en la interpretación que hacen sus críticos (Kliman, 2013).

\section{¿Qué postulan los temporalistas?}

A pesar de ser un caso paradigmático en la escuela marxista, ${ }^{7}$ Husson (cf. Perez, 1980) formula el primer trabajo de la interpretación temporalista, en el que esboza la necesidad de introducir el tiempo en el modelo marxista. Junto con el tiempo, el autor incluye dos apuntes críticos a los Neo-ricardianos: una formalización incoherente del ciclo del capital, y más importante, el uso del concepto de precio unitario derivado de la utilización de un modelo de estado estacionario, esto es, sin cambios en los precios de los inputs y los outputs. Como consecuencia, tendríamos un proceso de producción sin efecto temporal (Perez, 1980).

Los temporalistas recogen la idea de Husson y reclaman que la visión atemporal de los críticos de Marx implica ignorar que el valor individual de los inputs no tiene por qué coincidir con el valor social del output. Por lo tanto Bortkiewicz y los que aplican su solución a la transformación de valores en precios presentan el mismo error, al olvidar el factor tiempo, algo difícil de comprender si hablamos de modelizar la realidad de un proceso económico. Los autores temporalistas les recuerdan que a lo largo de un proceso productivo existe un desfase temporal entre la compra de los inputs, su posterior transformación y su comercialización y venta. De este desfase temporal surgirán diferencias en el valor del producto, que solo pueden venir explicadas por los incrementos de valor aportados por el capital vivo, el valor añadido por los trabajadores, como bien muestra Kliman (2007 y 2014).

Pero, ¿por qué los temporalistas inciden en que si existen diferencias de precios esto demuestra que existe un valor excedente y este ha sido añadido por los trabajadores? Primero, los temporalistas defienden la TVT, esto es, la idea de que el valor de una mercancía está determinado por el tiempo de trabajo incorporado en la misma, lo que a la vez implica que las ganancias de un capitalista estarán determinadas por el mayor número de horas de las que se puede adueñar, su Plusvalor. Esto supone que el constante cambio técnico al que está sometido el sistema de producción capitalista generará incrementos de la productividad y reducciones del trabajo por unidad de producto. Si se acepta esta premisa, ante una caída del precio de la mercancía por el aumento de la productividad, el valor incorporado también habrá caído.

A consecuencia de la caída del valor del trabajo en la mercancía, y a pesar de que la Masa de Ganancias total aumente, la Tasa de Ganancia resultará descendente. En tanto que para calcularla tenemos que la Tasa de Plusvalía se mantendrá constante (o con incrementos ínfimos) en el numerador, y en el denominador la COC aumentará por el incremento de capital

7 Hay que aclarar que en la bibliografía no aparecerá con su nombre, debido a que este trabajo donde expone sus primeras críticas a los Neo-ricardianos lo realiza bajo el sobrenombre de Manuel Perez. Cuando nos referimos a él como "paradigmático", es por su adhesión en sus trabajos posteriores a la New Interpretation, siendo crítico con algunos autores de la TSSI. 
constante (por la nueva maquinaria o la reducción del capital variable) respecto al variable. Así lo enuncia Astarita (2009):

Siguiendo a Marx [...] Dado que el cambio tecnológico es constante, los temporalistas afirman que al momento de consumirse productivamente, el valor del medio de producción ha bajado con respecto al momento en que fue adquirido, de manera que de conjunto el capital en proceso sufre una desvalorización lo que implica una baja de la tasa de ganancia. (Astarita, 2009, p. 5)

\section{La TSSI y los argumentos en favor de la consistencia de la Tasa de Ganancia}

Por tanto, hemos explicado en el apartado anterior cómo la introducción del tiempo permite enlazar la Teoría del Valor marxista con la caída de la Tasa de Ganancia, y a la vez eliminar la inconsistencia generada por la interpretación fisicalista y simultánea. ${ }^{8}$

Por otra parte, tendríamos el TO (Teorema de Okishio), que aparece en gran parte de los trabajos de los temporalistas, y al que en los noventa formulan una réplica, basada en los principios presentados anteriormente. Plantean que Okishio, como simultaneista, comete varios errores: primero, al formular un modelo de estática comparativa junto con la premisa de la igualación de los precios del input y el output, invalida su Teorema, al partir de un modelo económico estático sin factor tiempo. Estas premisas no se ven sustentadas en la realidad, en la cual encontramos un sistema económico donde los precios cambian rápidamente y el sistema presenta una inestabilidad periódica (Kliman, 2007).

El segundo problema que determinados autores señalan, entre ellos Naples (1989) y Freeman y Carchedi (1995), es el punto de partida de Okishio. Observan que sus premisas y supuestos (sin TVT, capital fijo constante y diferente modelización de la elección de tecnología) son completamente diferentes de los propuestos por Marx, lo que hace fácil llegar a unas conclusiones diferentes. Para estos autores, no se puede refutar a un autor sin respetar los supuestos iniciales claves que este realiza. Es Carchedi (1991) quien señala que Marx y Okishio presentan un objetivo diferente para el capitalista: "Okishio starts from the premise that capitalist introduce new techniques not necessarily because these techniques save labour (the productivity criterion) but because they reduce cost (the cost criterion)" (Carchedi, 1991, p.140). Es decir, Okishio parte de unos microfundamentos equivocados, o al menos no pueden ser denominados marxistas. Así mismo, Carchedi afirma que es cierto que la reducción de costes es importante para los empresarios pero lo es más el incremento de los beneficios como fin último.

8 Los simultaneistas, del término utilizado en inglés,simultaneist, son los autores que recurriendo a un modelo de estática comparativa sostienen que los precios y los valores son iguales, y por tanto su cálculo redundante, y utilizan el supuesto de que se mantienen sin alterarse a lo largo del periodo productivo.

Los fisicalistas, del término inglésphysicalist, lo utilizan los autores temporalistas para referirse a aquellos otros académicos dentro de los economistas radicales que aceptan las críticas a Marx, y que por lo tanto se caracterizan por desechar la TVT. Concluyen que la mejor manera de calcular la Tasa de Ganancias es en términos físicos, lo que implica que la Tasa de Ganancia no será negativa, y no sería esta la Teoría más adecuada para la explicación de las crisis capitalistas.

tiempo\&economía

Vol. $3 \mathrm{~N}^{\circ} 1$ - I semestre de 2016

p. 49 
En resumen, los temporalistas añaden la variable temporal contra las interpretaciones fisicalistas y simultaneistas, que en estos autores se encuentran claramente influidas por el walrasianismo de Bortkiewicz, así como por la idea de un equilibrio estático entre el momento previo a la aplicación de la mejora tecnológica y la situación posterior. Esto implicaría que cualquier mejora tecnológica conlleva una cantidad mayor producida, conclusión a la que se llega tras aceptar la invalidez de la TVT, lo que aleja a esta propuesta de la Teoría Marxista, pero sin elaborar una teoría alternativa alguna sobre el funcionamiento del sistema capitalista que permita comprender la recurrencia de sus crisis.

\section{Críticas recientes a la TSSI}

Frente a los planteamientos innovadores de la TSSI, tanto simultaneistas como fiscalistas han elaborado una serie de trabajos como respuesta, que permiten la consolidación de un amplio debate en torno a la Tasa de Ganancia, que desde los años noventa ha devuelto a la economía marxista aun primer plano dentro de las publicaciones heterodoxas.

Entre el primer grupo de críticas estarían las que acusan a los temporalistas de dogmáticos. Husson (2009) y Mongiovi (2009) argumentan que los temporalistas adolecen de una visión basada en la creencia que no tiene soporte argumentativo. De esta forma, Mongiovi (2009) sostiene que los temporalistas presentan el argumento del uso de la categoría economía burguesa para eliminar del debate las posiciones contrarias a Marx, aislando a su teoría de toda crítica. Otros trabajos simultaneistas, como los de Mohun (2003) y Mohun y Veneziani (2009), reclaman a los autores de la TSSI el abuso de los análisis hermenéuticos. Estos análisis se completan con la calificación de inconsistencia por parte de Mongiovi (2009) y Mohun (2003) al sistema teórico de los temporalistas, por estar basado en un sistema indeterminado.

Por otra parte, Heinrich (2013), en su reciente investigación sobre los nuevos textos de Marx recuperados por la Marx-Engels-Gesamtausgabe (MEGA), resalta la incapacidad de los autores temporalistas de apreciar e interiorizar que los problemas en la LTDTG surgen de la forma y el desarrollo de la construcción de la obra de Marx. Mohun (2003) afirma que gran parte de los supuestos de la TSSI están basados en las evidencias textuales del Segundo y Tercer Libro, compuestos por fragmentos inacabados por Marx. Y que, en cambio, no toman evidencias textuales del Primer Libro, que fue el único revisado y completado por el autor alemán. Esto, según Mohun, lleva a la incoherencia en gran parte de los análisis temporalistas. En esta línea, sostiene Heinrich (2013) que Marx no estaba conforme con la LTDTG y que nunca llegó a completar una teoría de las crisis, al faltarle, según él, tiempo para sumarle sus últimas investigaciones sobre el sistema financiero. Heinrich (2013) da un paso más a la hora de concluir sobre el Libro III de El capital y el Capítulo de la LTDTG, cuando sostiene que Marx no escribió completamente este capítulo, completando solo en sus notas las partes de la "ley como tal" y "las contratendencias de la ley". Por tanto, las conclusiones sobre las contradicciones internas de la Ley fueron realizadas por el editor: F. Engels.

Un segundo grupo de críticas incide en la invalidez de la TVT. La mayoría de estas apreciaciones se formulan sobre conceptos concretos, que son erróneos al ser definidos de la forma temporalista. Como afirman Mongiovi (2009) y Mohun y Veneziani (2009), la Teoría 
del Valor expuesta por los temporalistas no es más correcta que otras interpretaciones, como puede ser la de la New Interpretation (NI). Este grupo de autores continúa sosteniendo que es redundante utilizar el cálculo de los valores y de los precios, e insiste, rechazando claramente la TVT, en que las ganancias no solo dependen del tiempo de trabajo que pueden extraer los capitalistas (Mongiovi, 2009).

Este elenco de economistas cuestiona la coherencia de la metodología empleada por los marxistas temporalistas en la resolución del problema de la transformación y en la consistencia de la definición que estos proveen de la $\mathrm{MELT}^{9}$ (Veneziani, 2004; Mohunet al., 2006). Mohun et al. (2009) resumen los problemas teóricos de los temporalistas, distinguiendo entre problemas metodológicos y problemas de lógica. Entre los primeros esgrimen sus discrepancias sobre la posibilidad de sostener todos los argumentos presentados por autores como Kliman o Freeman, con base en los textos de Marx, por lo que no comparten la posición de que sostener la inconsistencia de la TSSI sea equivalente a afirmar la inconsistencia de la teoría marxista.

Además, los autores de la NI, representados por los trabajos de Dumenil y Levy, afirman que la TVT no conforma un marco suficiente para comprender los desequilibrios y las dinámicas del capitalismo, lo que no permite validar por sí sola la LTDTG, pero tampoco rechazarla. Estos mismos autores sostienen que son los salarios de los trabajadores los que finalmente definen el valor y los precios, posicionándose entre las interpretaciones fisicalistas (Kim, 2010).

Un tercer grupo de críticas a la TSSI estaría conformado por los planteamientos continuistas delTO, entre los que se encuentran los esbozados por dos de los autores marxistas más prolijos, Dumenil y Levy (2011), quienes retoman la polémica sobre la capacidad de los capitalistas de elegir voluntariamente o no la tecnología. Sostienen que si fuera una elección voluntaria, como Okishio defendía, la conclusión de que el cambio tecnológico produce en el largo plazo una caída sostenida de la Tasa de Ganancia estaría en contra de toda lógica. No obstante, rechazan la importancia que Okishio les otorgaba a los salarios como el único elemento capaz de hacer caer la Tasa de Ganancia, que refuta la visión de la Profit Squeeze.

Laibman es uno de los autores que, dentro de la controversia sobre el TO, ha aportado más argumentos a su favor. Laibman (1999) no acepta que con la mejora tecnológica los precios del producto (outputs) puedan ser menores que los pagados por los insumos y capitales (inputs), como fruto del abaratamiento temporal. Además defiende la contabilidad "material" de la Tasa de Ganancia, posicionándose frente a la contabilidad de los costes sustentada por los temporalistas, o historical cost. Así, Laibman (2001) entiende que los autores de la TSSI solo utilizan esta contabilidad, sea la correcta o no, porque les permite cumplir la LTDTG: "To put the matter in the simplest posible terms: the temporalist claims fail. The Okishio Theorem is true" (Laibman, 2001, p. 91).Por último, Laibman insiste en la cuestión del efecto amortiguador de las causas contrarrestantes, que ya vimos en las críticas a Marx en los sesenta y setenta.

9 MELT es la expresión monetaria del trabajo (en inglés, Monetary Expression of Labor Time), la cual permite solventar los problemas con el actual papel fiduciario como moneda de intercambio. Para una mejor comprensión de la MELT recomiendo la lectura del trabajo de Mosley (2011).

tiempo\&economía

Vol. $3 \mathrm{~N}^{\circ} 1$ - I semestre de 2016

p. 51 


\section{La respuesta temporalista a sus críticos}

En los últimos años, los trabajos de los autores temporalistas como Kliman, Freeman y Roberts han estado dirigidos a dar respuesta a las críticas formalizadas en contra de esta nueva interpretación, resumidas en el apartado anterior. El propio trabajo de Kliman (2007) es un claro ejemplo, sin duda la mejor síntesis sobre el planteamiento temporalista y su confrontación con las interpretaciones que alegan la inconsistencia de la Teoría de Marx.

Actualmente, uno de los debates que sigue abierto por la complejidad teórica del mismo se encuentra en torno a las distintas formalizaciones de la MELT. La no implicación de este trabajo en la cuestión monetaria permite dejarlo al margen. No obstante, hay que resaltar sus importantes implicaciones en la disciplina económica, tanto dentro de la macroeconomía como de la política monetaria, pues su existencia es contrapuesta a la teoría monetarista estudiada en las facultades de Economía (Kim, 2010). ${ }^{10}$

El trabajo de Heinrich (2013)ha sido uno de los que más réplicas temporalistas ha acumulado. Entre las numerosas críticas formuladas por Heinrich estaría la insistencia en la indeterminación de la dirección de la Tasa de Ganancia respecto al rol de la tasa de plusvalía en la ecuación. Este autor sostiene que la plusvalía puede crecer más que la COC, crítica rebatida por Carchedi y Roberts (2013), quienes aceptan la posibilidad de realizar ejemplos numéricos en los cuales la Tasa de Ganancia crezca en todo momento por la acción de la Tasa de Plusvalía. Sin embargo, tales representaciones serán formalizaciones matemáticas sin ningún sustento en la economía real, puesto que en esta se observan, para distintos momentos temporales, caídas en la Tasa de Ganancia, a las que se añade la existencia de barreras legales y biológicas en la jornada de trabajo, que imposibilitan la extensión de la Plusvalía más allá de unos límites (Kliman, Freeman, Potts, Gusev y Cooney, 2013).

Un error determinante en la argumentación de Heinrich, que otros autores también han cometido, está en su interpretación de los textos originales de Marx, al confundir y modificar lo escrito por él. Por ejemplo, al cambiar la formulación de la LTDTG y traducir la expresión mustfall ("debe caer", esto es, con un sentido de obligación), enunciando la Ley como un suceso seguro. Sin embargo, Marx utilizó (traducido al inglés) willfall ("caerá", como suceso que acontecerá, sin sentido de obligación) o tend to fall ("tiende a caer"), como un suceso posible. Heinrich pretende utilizarlo como una prueba del error de Marx, pero realmente es un fallo del autor por malinterpretar el texto (Kliman et al., 2013), aclarando que la tendencia a descender de la Tasa de Ganancia, aunque no se presentase siempre bajo todas las circunstancias, no prueba nada: "Heinrich's mistaken belief that Marx sought to prove that the rate of profit must fall in the long run, under all circumstances, is intimately bound up with his failure to understand the purpose and function of the LTFRP" (Kliman et al., 2013, p.4). ${ }^{11}$

10 El tema puede seguirse, como se ha dicho en la nota 9. Tema inabordable en este artículo por estar alejado del objetivo y extensión del mismo.

11 Aun es más problemática la afirmación de Heinrich al no citar ningún texto de Marx. Esto impide otra evaluación que no sea de los textos publicados de Marx, en los que Kliman et al. (2013) encuentran todo lo contrario a lo sustentado por Heinrich: "He supplies no citation here, and Marx's text does not suppor this claim. In particular, the following statement directly contradicts it". 


\section{¿CUÁL ES EL SOPORTE EMPÍRICO DE LA LTDTG?}

Al margen de los debates teóricos, siempre ha existido una crítica, realizada desde los enfoques más ortodoxos de la economía, acerca de la validez de estudios empíricos de las teorías marxistas. Frente a esta demanda, es precisamente esta nueva interpretación la que aporta un importante trabajo empírico en la última década, donde ha conseguido obtener datos sobre la Tasa de Ganancia, la Tasa de Plusvalía, la Tasa de Acumulación y la COC, que les permiten avanzar en los análisis sobre las crisis y los ciclos en el capitalismo.

Estos análisis empíricos no están exentos de problemas. Entre los que se destaca la dificultad de compatibilizar las categorías marxistas con la contabilidad de los países capitalista; de ahí que gran parte de los autores realicen sus trabajos sobre el caso nacional de EE. UU.12:"Most empirical work up to now has been concentrated on measuring the US rate of profit and trying to get a measure that is close as possible to Marxist categories, i.e. à la Marx" (Roberts, 2012, p.2), debido a la importante base de datos que se puede encontrar sobre las cuentas nacionales de este país.

La importancia de la actual crisis ha venido de la mano de la realización, por parte de los autores marxistas, de un buen número de trabajos en los que se ha podido probar la capacidad explicativa de la LTDTG, entre los que podemos destacar los de Valle (2008), Freeman (2009), Izquierdo (2014),Harman (2010), Carchedi (2011), Roberts (2012), Kliman (2012) y Carchedi y Roberts (2013), desde la óptica temporalista. Y desde la NI, los trabajos de Husson (2010), Tapia y Astarita (2011), y Dumenil y Levy (2011). Si bien es cierto que no todos ellos caracterizan la crisis actual como una crisis resultante de la caída de la Tasa de Ganancia, al menos todos ellos sí observan una caída de la Tasa de Ganancia en los años anteriores al crash financiero de 2009. Coincidiendo entre los temporalistas, sus trabajos puntualizan en 1965-1968 el momento de mayor rendimiento del capital desde el final de la Segunda Guerra Mundial; con la excepción de dos autores, Freeman (2009) y Kliman (2012), quienes sostienen que la caída tendencial comenzó al finalizar el conflicto bélico y no ha dejado de caer, siendo esta caída causada por el crecimiento sostenido de la COC., hecho que Carchedi y Roberts (2013) parecen comprobar:

To mention only one example, in the productive sectors of the US economy, the number of labourers per one million of (deflated) dollars of assets has fallen persistently from 65 in 1947 to 6 in 2010. (Carchedi y Roberts, 2013, p. 2)

De forma más acotada, antes de la crisis actual, Carchedi y Roberts (2013) encuentran que para EE. UU., entre 1997 y 2008, la Tasa de Ganancia cayó un 12\%, a la vez que COC crecía un 22\%; en cambio, la Plusvalía creció únicamente un $2 \%$. Esto demuestra empíricamente todos los pasos que caracterizan la LTDTG, con un crecimiento de la COC, un incremento o estancamiento de la Plusvalía como consecuencia de los límites que presenta, y por último, una caída en el largo plazo de la Tasa de Ganancia. Además, Carchedi y Roberts (2013), Freeman

12 No obstante, existen diferentes trabajos para otros países que se pueden consultar, como los ejemplos de Minqui Li y Zhi Li (2015) sobre China, y Roberts (2012) sobre Europa, principalmente Inglaterra. Para el caso español, los trabajos más destacados son los de Izquierdo(2007) y Guerrero (2006), y los de Dumenil y Levy (2001), sobre Francia.

tiempo\&economía

Vol. $3 \mathrm{~N}^{\circ} 1$ - I semestre de 2016

p. 53 
(2009) y Kliman (2012) observan que la capacidad explicativa de la COC respecto al descenso de la Tasa de Ganancia estaba en torno al 60\%. De manera similar, Izquierdo (2014) encuentra que cerca del 78\% de la caída de la Tasa de Ganancia en dos periodos distintos (entre 1928-1973 y 1974-1983) se explicaría por el incremento de la productividad. No hay que olvidar que no se identifica el inicio de la crisis únicamente con una caída prolongada de la Tasa de Ganancia, sino cuando esta provoca un estancamiento o caída de la Masa de Ganancias. Precisamente esto es lo que Carchedi (2011) y Kliman (2012) encuentran para EE.UU. entre 2006 y 2009, con una caída de 10 puntos porcentuales.

Dentro de la óptica marxista se reconoce otro argumento sostenido por autores como Husson (2010), Tapia y Astarita (2011), Dumenil y Levy (2011): que, a pesar de que en momentos puntuales se observen caídas de la Tasa de Ganancia, no todas las crisis son consecuencia de esta variable. Husson (2010) y Dumenil y Levy (2011) plantean que la principal causa de la actual crisis es el cierre del ciclo de las políticas neoliberales, entendido como un nuevo estadio del capitalismo, que imprimieron una fuerza explotadora al capital sobre el trabajo desde los setenta, pero que acabaron con una ruptura del crédito con el que habían sostenido la demanda de los trabajadores. Fue, por tanto, la ruptura del consumo lo que en última instancia llevó a la inestabilidad de la economía, y no la caída de la Tasa de Ganancia.

Por tanto, ambos grupos de autores, tanto temporalistas como los que representan la NI, encuentran en los datos una caída en la Tasa de Ganancia anterior a la crisis. Sin embargo, discrepan en la causa última de esta: los temporalistas alargan la tendencia desde los cuarenta o los sesenta y, por lo tanto, la presentan como causa final de la crisis, mientras que los autores de la NI no aprecian una tendencia en el largo plazo y formulan una teoría sobre la crisis basada en el poder financiero y la caída del consumo endeudado.

\section{CONCLUSIONES}

Este trabajo pretendía situar el actual estado de los debates en torno a la Tasa de Ganancia dentro de la teoría marxista y analizar la consistencia de la LTDTG, definida por Marx como teoría explicativa de las crisis. Para lograr esto se introdujo una nueva interpretación de esta Teoría, la temporalista, que presenta diferentes argumentos para la defensa de la consistencia de la Teoría de Marx sobre las crisis, sosteniendo que los problemas de incoherencia no se encuentran en la Teoría formulada por el filósofo alemán, sino en la interpretación que hacen los autores críticos de la misma.

Concretamente, los autores temporalistas señalan dos errores en las interpretaciones críticas respecto a Marx:

- Primero, la formulación de una teoría marxista sin la base de la TVT, lo que equivale a eliminar la teoría de la explotación, es decir, suprimir la base del cuerpo teórico de Marx.

- Segundo, la utilización de herramientas incompatibles con la visión dinámica que tenía Marx del sistema capitalista, puesto que estos autores no solo eliminan la importancia del valor, sino también el factor temporal del proceso de producción de mercancías. 
Se puede concluir que los autores de la TSSI han formulado la única interpretación consistente con la validación del análisis de Marx en el Libro III de El capital. Estos autores marxistas únicamente basan su trabajo en la introducción de la variable "tiempo" en el modelo de Marx, lo que les permite superar las críticas de inconsistencia dirigidas a la TVT y la LTDTG.

Si bien es cierto que los debates en torno a esta teoría y a la TVT no están ni mucho menos cerrados, es precisamente esta confrontación de argumentos e interpretaciones la que puede permitir avanzar a la Teoría económica marxista hacia un nivel que la adecue al menos a las demás teoría heterodoxas. Esto implicará que más autores se interesen por el estudio de esta corriente, desarrollando un mayor número de análisis sobre el cumplimiento de esta Ley en distintas crisis y para diferentes países, es decir, en distintos contextos.

Siendo esta última limitación uno de los mayores impedimentos para que la LTDTG pueda convertirse en una teoría para la explicación del capitalismo mundial, es decir, la dificultad de trabajar con algunas de las categorías marxistas, más en concreto, en los países donde no existen buenas bases de datos económicos.

En último término, este trabajo también ha resaltado la capacidad del enfoque marxista temporalista de aislar una causa determinante, que no única, de las crisis capitalistas, demostrando sus capacidades frente a los demás enfoques económicos. La mayoría de los demás enfoques sostienen una causalidad distinta, dependiendo de la variable o variables cuantificadas que reflejen la alarma en cada crisis (crisis financieras, crisis de tipo de cambio, crisis de demanda efectiva, crisis de la deuda, etcétera), siendo este uno de los mayores impedimentos para anticipar o prever las crisis económicas y sus nefastas consecuencias sociales.

\section{REFERENCIAS}

Astarita, R. (2009). El Teorema de Okishio y las crisis. Documento de trabajo. Recuperado de: http://rolandoastarita.wordpress.com/.

Astarita, R. (2011).ValorTrabajo, el Problema de la Transformación y crítica sraffiana. Documento de trabajo. Recuperado de: http://rolandoastarita.wordpress.com/.

Burawoy, M. (1990). Marxism as science: Historical challenges and theoretical growth. American Sociological Review, 55, 775-793. Recuperado de: http://burawoy.berkeley.edu/Marxism/ Marxism\%20As\%20Science.pdf

Carchedi, G. (1991). Frontiers of political economy. Londres: Verso.

Carchedi, G. (2011). Behind and beyond the crisis.International Socialism, 132. Recuperado de: http://isj.org.uk/behind-and-beyond-the-crisis/

Carchedi, G y Roberts, M. (2013). A critique of Heinrich's, crisis theory, the law of the tendency of the profit rate to fall, and Marx's studies in the 1870s, Monthly Review, 65(7). Recuperado de: http://monthlyreview.org/commentary/critique-heinrichs-crisis-theory-law-tendencyprofit-rate-fall-marxs-studies-1870s/ 
Dumenil, G. y Levy, D. (2001). The classical-marxian evolutionary model of technical change: Application to historicaltendencies.Paper presented in the URPE Session, The Classical Approach to Technical Change and Capital Accumulation, of the ASSA meetings, New York, January 3-5, 1999

Dumenil, G. y Levy, D. (2011). The crisis of the early $21^{\text {st }}$ century: A critical Review of Alternative Interpretations. Documento de Trabajo de CNRS. Recuperado de: http://www.jourdan.ens. fr/levy/dle2011e.pdf

Freeman, A. (2000, Junio). Marxian debates on the falling rate of profit. Greenwich: University of Greenwich, 29 páginas. Recuperado de: http://mpra.ub.uni-muenchen.de/2588/

Freeman, A. (2009). What makes the US rate of profit fall? Documento de trabajo. Recuperado de: http://mpra.ub.uni-muenchen.de/14147/

Freeman, A. y Carchedi, G. (eds.) (1995). Marx and non-equilibrium economics. Aldershot: Edward Elgar.

Freeman, A., Chick, V. y Kayatekin, S. (2014) Samuelson's ghosts: Whig history and the reinterpretation of economy theory. Cambridge Journal of Economist, 38(3), 519-529.

Gigliani, G. (2007, Diciembre). Tasa de ganancia y Macroeconomía. Buenos Aires, Argentina, I Jornada de Economía Política, Universidad Nacional de General Sarmiento.

Guerrero, D. (2006). La explotación de los asalariados y la ganancia capitalista en España (19542001). Razón y Revolución: teoría, historia, política, 16, 23-56.

Guerrero, D. (2008). Un resumen completo de El Capital de Marx. Madrid: Maia.

Harman, C. (2010). Not all Marxism is dogmatism: A reply to Michael Husson, International Socialism, 125. Recuperado de: http://isj.org.uk/not-all-marxism-is-dogmatism-a-reply-tomichel-husson/

Heinrich, M. (2013). Crisis theory, the law of the tendency of profit rate to fall, and Marx's studies in the 1870s. MonthlyReview,64(11). Recuperado de: http://monthlyreview.org/2013/04/01/ crisis-theory-the-law-of-the-tendency-of-the-profit-rate-to-fall-and-marxs-studies-in-the1870s

Husson, M. (2009). El dogmatismo no es un marxismo. Viento Sur, 107,75-84.

Husson, M. (2010). Le débat sur le taux de profit. Imprecor, 562-563.

Izquierdo, S. (2007). The Dynamics of the Profit Rate in Spain (1954-2001). Review of Radical Political Economics, 39, 543-561.

Izquierdo, S. (2014).The role of long-term and short-term dynamics of the US rate of profit in the current crisis. International Journal of Management Concepts and Philosophy, 8(1), 1-22.

Katz, C. (2010). La economía marxista, hoy: seis debates teóricos. Madrid: Maia Ediciones.

Kim, C. (2010). The recent controversy on Marx's value theory: A critical assessment. Marxism 21, 282-320.

Kliman, A. (2007). Reclaiming Marx's Capital. Plymouth: Lexington Books. 
Kliman, A. (2012). The failure of capitalist production: Underlying causes of the great recession. Londres: Pluto Press.

Kliman, A. (2013). A defense of pluralism. Documento no publicado. Recuperado de: http:// akliman.squarespace.com.

Kliman, A. (2014). The Whiggish foundations of Marxian and Sraffian economics. Cambridge Journal of Economist, 38, 643-661.

Kliman, A. y Freeman, A. (2006). Replicating Marx: A reply to Mohum. Capital and Class, 88, 117-123.

Kliman, A., Freeman, A., Potts, N., Gusev, A. y Cooney, B. The Unmaking of Marx's Capital: Heinrich's Attempt to Eliminate Marx's Crisis Theory (July 22, 2013). Recuperado de http: // ssrn.com/abstract=2294134. doi: http://dx.doi.org/10.2139/ssrn.2294134

Laibman, D. (1999). Okishio and His critics: Historical cost vs. replacement cost. Research in Political Economy, 17, 207-227.

Laibman, D. (2001). Rising 'material' vs. falling 'value' rates of profit: Trial by simulation. Capital\&Class, 73, 79-96.

Marx, K. (2007 [1894]). El capital: crítica a la economía política. Traducción Vol. 3. Madrid: Edición Akal.

Minqui Li y Zhi Li (2015) China's Falling Rate of Profit and the Coming Economic Crisis, Economic and Political Weekly, L(41), 27-31, October.

Mohun, S. (2003). On the TSSI and the exploitation theory of profit. Capital\&Class, 81, 85-102.

Mohun, S. y Veneziani, R. (2006). The incoherence of the TSSI. Capital \&Class, 31(2), 139-145.

Mohun, S. y Veneziani, R. (2009). The temporal single-system interpretation: Under determination and inconsistency. Marxism 21( 6), 277-301.

Mongiovi, G. (2009). Economía vulgar en ropaje marxista: una crítica del marxismo del Sistema Temporal Simple. Circus, año 2, 107-137

Mosley, F. (2011). The determination of the monetary expression of labour time (MELT) in the case of non-commodity money. Review of Radical Politics Economics, 43, 95-105.

Naples, M.I. (1989). A radical economic revision of the transformation problem. Review of Radical Political Economics, 21(1-2), 137-158

Perez, M. (1980). Valeur et prix: un essai de critique des propositions néo-ricardiennes. Critiques l'economie politique, nouvelle série n¹0 Janviers-Mars.

Roberts, M. (2012). A world rate of profit. Paper presentado en la Association of Heterodox Economists. Recuperado de: http://thenextrecession.wordpress.com

Robinson, J. (1968). Introducción a la economía marxista. Madrid: Siglo XXI.

Shaikh, A. (1990). Valor, acumulación y crisis. Bogotá: Tercer Mundo Editores.

Tapia, J. A. y Astarita, R. (2011). La Gran Recesión y el capitalismo del siglo XXI. Madrid: Catarata.

Valle, A (2008). La crisis estadounidense y la ganancia. Razón y Revolución, 18, 79-93.

tiempo\&economía 
Veneziani, R. (2004). The temporal single-system interpretation of Marx's economics: A critical evaluation. Metroeconomica, 55(1), 96-114. 